\title{
Automatic Statistical Identification of Neuroanatomical Abnormalities between Different Populations
}

\author{
Alexandre Guimond ${ }^{1}$, Svetlana Egorova ${ }^{1}$, Ronald J. Killiany ${ }^{2}$, \\ Marilyn S. Albert ${ }^{3}$, and Charles R. G. Guttmann ${ }^{1}$ \\ ${ }^{1}$ Center for Neurological Imaging, Brigham and Women's Hospital, Harvard Medical School \\ \{guimond, egorova, guttmann\}@bwh. harvard.edu \\ 2 Department of Anatomy and Neurobiology, Boston University \\ rkilliany@cajal-1.bu.edu \\ ${ }^{3}$ Departments of Phychiatry and Neurology, Massachusetts General Hospital, Harvard Medical \\ School, albert@psych.mgh.harvard.edu
}

\begin{abstract}
We present a completely automatic method to identify abnormal anatomical configurations of the brain resulting from various pathologies. The statistical framework developed here is applied to identify regions that significant differ from normal anatomy in two groups of patients, namely subjects who subsequently converted to Alzheimer's Disease (AD) and subjects with mild AD. The regions identified are consistent with post-mortem pathological findings in AD.
\end{abstract}

\section{Introduction}

An important tool used to diagnose abnormal anatomical variations are medical atlases [1]. Traditional ones, such as by Talairach \& Tournoux [2] or Schaltenbrand \& Wahren [3], are presented in textbooks, but computerized atlases comprising information in a more practical and quantitative manner are becoming available [4-16]. They usually include information obtained from a set of subjects, as opposed to a single individual in most paper atlases, making them more representative of a population. For example, the Montreal Neurological Institute used 305 normal subjects to build an atlas comprising intensity variations after affine registration in the stereotaxic space defined by Talairach \& Tournoux [8]. These methods also enable the calculation of normal shape variations [17-19].

Still, most of the clinical work regarding the identification of abnormal brain anatomy due to particular diseases involves manual intervention by skilled anatomists [20, 19]. The following work aims at developing a fully automated method that identifies regions in which the anatomy is significantly different between two given populations. We describe a statistical framework to perform such an analysis based on statistics obtained from displacement fields resulting from deformable registration. In the following section we describe the method developed to identify such regions. The groups of subjects used to test our procedure are then detailed, followed by results and a brief discussion.

\section{Methodology}

Affine registration based on the correlation ratio [21,22] is performed between the images from the groups under study and a reference image. Deformable registration based 
on the demons' algorithm [23, 24], an intensity based registration method, is then performed to assess non-linear anatomical variations present between the reference image and each image of the two groups. The displacement fields resulting from the deformable registration are then used to assess significant anatomical differences between the groups of subjects. The statistical analysis performed to identify these differences follows.

Let $u_{i j}(x)$ be the three-dimensional displacement found by deformable registration at an anatomical location $x$ in the reference scan to the corresponding anatomical location in image $j$ of group $i$. Two important statistics that can be computed from these displacements are the groups' sample mean vector $\bar{u}_{i}(x)$ and covariance matrix $S_{i}(x)$,

$$
\begin{aligned}
& \bar{u}_{i}(x)=\frac{1}{N_{i}} \sum_{j=1}^{N_{i}} u_{i j}(x), \\
& S_{i}(x)=\frac{1}{N_{i}-1} A_{i},
\end{aligned}
$$

where $N_{i}$ is the number of subjects in group $i$, and $A_{i}$ is the matrix of sums of squares and cross products of deviations about the mean for group $i$,

$$
A_{i}=\sum_{j=1}^{N_{i}}\left[u_{i j}(x)-\bar{u}_{i}(x)\right]\left[u_{i j}(x)-\bar{u}_{i}(x)\right]^{\prime} .
$$

The following aims at identifying significant anatomical differences due to shape variations of the various structures composing the human brain. This will be done by assessing significant differences between the average displacements $\bar{u}_{i}(x)$ found for each group. Assuming that the displacements found for group $i\left(u_{i 1}(x), \ldots, u_{i N_{i}}(x)\right)$ are samples from a normal distribution $N\left(\mu_{i}, \Sigma_{i}\right)$, two common tests based on the $T^{2}$ statistic to assess whether the mean of one population $a$ is equal to the mean of another population $b$ are available. The use of one test or the other depends on the equality of the covariance matrices of each population.

In the case that both populations have the same covariance $\left(\Sigma_{a}=\Sigma_{b}\right)$, it can be shown [25] that

$$
\frac{N_{a} N_{b}\left(N_{a}+N_{b}-4\right)}{3\left(N_{a}+N_{b}\right)\left(N_{a}+N_{b}-2\right)}\left[\bar{u}_{a}(x)-\bar{u}_{b}(x)\right]^{\prime} S^{-1}\left[\bar{u}_{a}(x)-\bar{u}_{b}(x)\right],
$$

where

$$
\begin{aligned}
& S=\frac{1}{N_{a}+N_{b}-2} A, \\
& A=A_{a}+A_{b},
\end{aligned}
$$

follows an $F$ distribution with 3 and $N_{a}+N_{b}-4$ degrees of freedom.

If both populations have different covariances $\left(\Sigma_{a} \neq \Sigma_{b}\right)$, another test is used [25]. In this case, assuming without loss of generality that $N_{a} \leq N_{b}$,

$$
\frac{N_{a}\left(N_{a}-3\right)}{3\left(N_{a}-1\right)}\left[\bar{u}_{a}(x)-\bar{u}_{b}(x)\right]^{\prime} S^{-1}\left[\bar{u}_{a}(x)-\bar{u}_{b}(x)\right]
$$


where

$$
\begin{aligned}
S & =\frac{1}{N_{a}-1} \sum_{j=1}^{N_{a}}\left[y_{j}(x)-\bar{y}(x)\right]\left[y_{j}(x)-\bar{y}(x)\right]^{\prime}, \\
y_{j}(x) & =u_{a j}(x)-\sqrt{N_{a} / N_{b}} u_{b j}(x), \quad j=1, \ldots, N_{a}, \\
\bar{y}(x) & =\sum_{j=1}^{N_{a}} y_{j}(x),
\end{aligned}
$$

follows an $F$ distribution with 3 and $N_{a}-3$ degrees of freedom.

When comparing displacement fields from populations that are afflicted by different diseases, assuming the equality of covariance matrices might not be the proper choice. To answer this question, we use another criterion designed to test the equality of different covariance matrices. By defining

$$
\lambda=\left[\left(\frac{1}{k_{a}}\right)^{k_{a}}\left(\frac{1}{k_{b}}\right)^{k_{b}}\right]^{\frac{3}{2} n} \frac{\left|A_{a}\right|^{\frac{1}{2} n_{a}}\left|A_{b}\right|^{\frac{1}{2} n_{b}}}{|A|^{\frac{1}{2} n}},
$$

where $n_{a}=N_{a}-1, n_{b}=N_{b}-1, n=n_{a}+n_{b}, k_{a}=n_{a} / n$, and $k_{b}=n_{b} / n$, it can be shown [25] that the asymptotic expansion of the distribution of $-2 \rho \log \lambda$ is

$$
\operatorname{Pr}\{-2 \rho \log \lambda \leq z\}=\operatorname{Pr}\left\{\chi_{6}^{2} \leq z\right\}+\omega\left[\operatorname{Pr}\left\{\chi_{10}^{2} \leq z\right\}-\operatorname{Pr}\left\{\chi_{6}^{2} \leq z\right\}\right]+O\left(n^{-3}\right),
$$

where

$$
\begin{aligned}
& \rho=1-\frac{13}{12}\left(\frac{1}{n_{a}}+\frac{1}{n_{b}}-\frac{1}{n}\right), \\
& \omega=\frac{5\left(\frac{1}{n_{a}^{2}}+\frac{1}{n_{b}^{2}}-\frac{1}{n^{2}}\right)-3(1-\rho)^{2}}{2 \rho^{2}} .
\end{aligned}
$$

Note that for a large $n$ or for large values in $A$, the power calculations for the determinants of Eq. 11 will create overflows on most computers. To solve this problem, we identify the whitening transformation [26] for $A$ and apply it to $A, A_{a}$, and $A_{b}$ prior to calculating Eq. 11. Although this transformation changes the matrices, since the same linear transformation is applied to all of them, there is no effect on the result of the criterion of Eq. 11.

The whitening transformation for $A$ is $\Phi \Lambda^{-1 / 2}$, where $\Phi$ is the matrix of eigenvectors of matrix $A$ and $\Lambda$ is the diagonal matrix consisting of its eigenvalues. This transformation makes $A$ the identity matrix and the power calculations for the determinants of $A_{a}$ and $A_{b}$ fall within range on our computers ${ }^{4}$. Using this method, Eq. 11 becomes:

$$
\lambda=\left[\left(\frac{1}{k_{a}}\right)^{k_{a}}\left(\frac{1}{k_{b}}\right)^{k_{b}}\right]^{\frac{3}{2} n}\left|\tilde{A}_{a}\right|^{\frac{1}{2} n_{a}}\left|\tilde{A}_{b}\right|^{\frac{1}{2} n_{b}},
$$

\footnotetext{
${ }^{4}$ Although this strategy was sufficient for our purposes, for very large $n(n \gg 200)$ the same problem will occur and other strategies will have to be explored.
} 
where

$$
\begin{aligned}
\tilde{A}_{a} & =\left[\Phi \Lambda^{-1 / 2}\right]^{\prime} A_{a}\left[\Phi \Lambda^{-1 / 2}\right], \\
\tilde{A}_{b} & =\left[\Phi \Lambda^{-1 / 2}\right]^{\prime} A_{b}\left[\Phi \Lambda^{-1 / 2}\right] .
\end{aligned}
$$

Using Eq. 15, we can calculate $p$-value maps (or $p$-maps) that correspond to the probability that the covariance of the displacements from any two groups $a$ and $b$ are equal at a given anatomical position $x$ in the reference scan. By setting the significance level at 0.95, and thus rejecting the null hypothesis that the covariances are equal for $p$-values smaller than 0.05 , we can assess whether there is sufficient evidence from the displacements found at $x$ for groups $a$ and $b$ to assume that the covariance of the displacements for both groups are different at that point.

If we accept the null hypothesis (the covariances from both groups are equal), Eq. 4 is used to test whether the mean displacement from both groups are equal. If the null hypothesis is rejected, Eq. 7 is used to perform the same test. In either case, we set the significance level at 0.95 . This process is performed for each voxel $x$ of the reference scan to identify anatomical position where there is significant evidence showing different average displacement between the two groups. In practical terms, this means that brain anatomy at position $x$ for the two groups differs. If one of the groups is the normal group, these results show strong evidence that the identified regions correspond to pathological variations due to the disease affecting the other group.

\section{Data}

Detailed information regarding the subjects and data used in this study can be found in [20]. Briefly, the subjects in this study consisted of elderly individuals. All subjects were 65 or older, free of significant underlying medical, neurological, or psychiatric illness, and meet the Clinical Dementia Rating (CDR) [27] criteria described below. The CDR scale was designed to stage individuals according to their functional ability, from 0 representing normal function, to 5 representing the terminal phase of dementia.

A total of 74 subjects were used in this study. At the time of the MR acquisition, 37 of them were identified as normals $(\mathrm{CDR}=0), 20$ as "questionable $\mathrm{AD}$ " $(\mathrm{CDR}=0.5)$ who converted to AD according to clinical research criteria for probable AD [28] after a 3 year follow-up, and 16 as "mild AD" according to the same criteria $(\mathrm{CDR}=1.0)$. These 3 groups will be referred to as normals, converters, and mild AD respectively in the following. Another elderly subject identified as normal $(\mathrm{CDR}=0)$ was also used and served as a reference image for the following work.

The MRI data used in this study consisted of three-dimensional T1-weighted gradient echo scans of the brain $\left(\mathrm{TR}=35 \mathrm{~ms}, \mathrm{TE}=5 \mathrm{~ms}, \mathrm{FOV}=240 \mathrm{~cm}^{2}\right.$, flip angle $=45^{\circ}$, slice thickness $=1.5 \mathrm{~mm}$, matrix size $=256 \times 256$ ). 124 coronal slices covering the entire brain were acquired for each subject. The resulting data volumes were composed of $256 \times 256 \times 124$ voxels, each of size $0.9375 \times 0.9375 \times 1.5 \mathrm{~mm}^{3}$. 


\section{Results and Discussion}

$p$-maps were generated as described in the previous section to identify anatomical locations where brain anatomy between two groups of subjects differed. In Fig. 1-a, we present regions identified as significantly different $(\mathrm{p}=0.05)$ between the normal and converter groups. Fig. 1-b, presents the result of the same test using the normal and mild AD groups. Of particular interest are the larger regions which lie in the inferior parietal lobule region (arrow in Fig. 1-a) and close to the hippocampal area (arrow in Fig. 1-b). These regions of the association and heteromodal cortex are known to be strongly affected by the characteristic plaques and tangles in AD [29]. Fig. 1-c shows a 3D rendering of pathological regions as identified by our method for the converter group. Note that most small regions lie inside the grey matter, in the posterior half of the brain, where grey matter atrophy has been reported for AD patients [29].

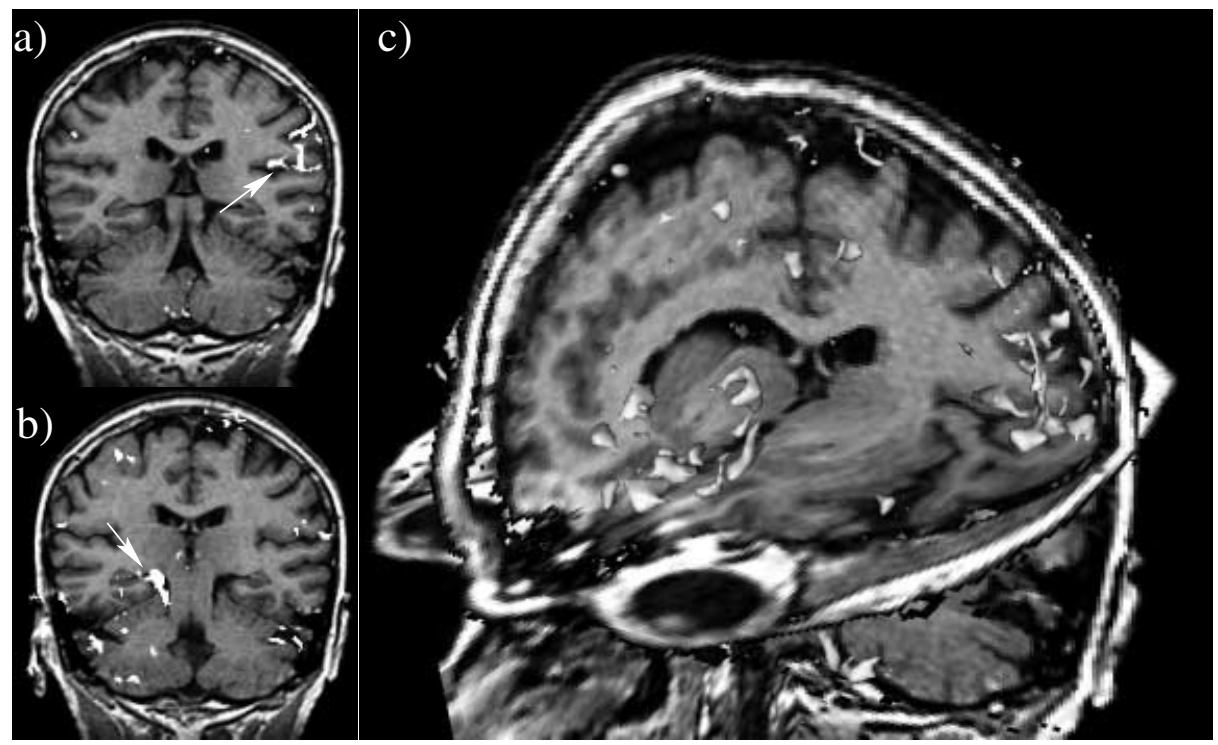

Fig. 1. Pathological regions as identified by our method between a) normal controls and patients who converted to AD 3 years after the MRI was performed, and b) normal controls and patients with mild AD. Also shown in c) is a $3 \mathrm{D}$ rendering of pathological regions (in red) as identified by comparing the normal and converter groups.

Although the statistical methods presented here to analyze displacements are independent of the registration method used, the confidence we have in the regions identified as pathological is based on the assumption that registration provided appropriate correspondence fields between the anatomical locations of the reference image and subjects under study. As mentioned previously, our deformable registration method is intensitybased, and as for most registration methods, one underlying assumption is the equivalence of brain topology between the images registered. As displayed in Fig. 2, this is not 
strictly the case when registering our reference image with AD patients. In this figure, we show corresponding coronal slices from the T1-weighted images of the reference subject (Fig. 2-a) and an AD patient (Fig. 2-b). These images have been processed to emphasize the contrast inside the white matter. As can be seen, the white matter of the reference subject is noisy, but represents a relatively uniform intensity region. In contrast, the white matter of the AD patient is much less uniform, with white matter signal abnormalities present at many locations (See arrows in Fig. 2-b). These abnormalities will tend to shrink to small regions during registration and the resulting displacement field will reflect this phenomenon. Hence, the statistics obtained from the displacement are encoding information regarding displacements that are due to brain shape differences, as well as white matter anomalies. Whether this is desired or not when building statistical brain models is probably disease and application dependant, and is currently under investigation in our group. A solution to solve a similar problem for multiple sclerosis patients is presented in [30].
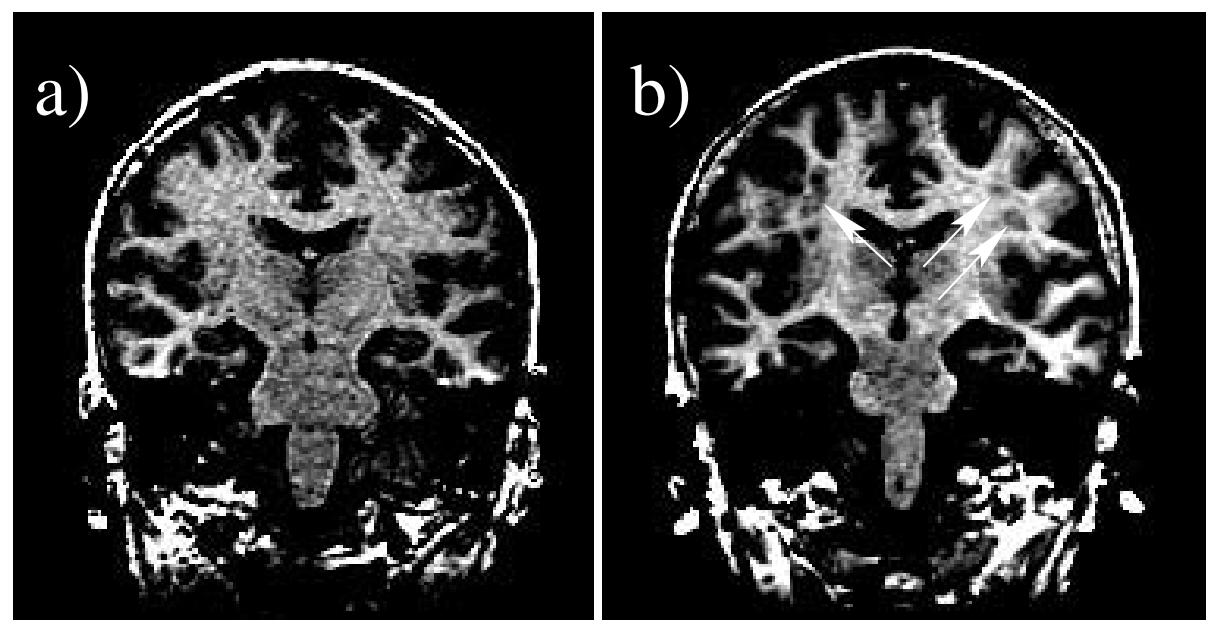

Fig. 2. T1-weighted MR images where contrast has been emphasized in the white matter: a) an elderly normal subject (the reference subject) and b) a patient with mild AD. Note the white matter signal abnormalities present in the patient (See arrows) but not in the reference subject.

Another aspect we are currently investigating is the incorporation of spatial information to compute the statistical maps produced with our method. The analysis presented here works on a voxel per voxel basis and does not take into account any information from neighboring voxels, but it is likely that information concerning abnormalities of neighboring voxels should be taken into account to calculate the $p$-value for a given anatomical position. To this end, we are currently investigating the incorporation of Markov Random Fields into our model in a way similar to that described in [31]. 


\section{Conclusion}

We have presented a statistical framework to analyze displacement fields obtained from deformable registration and identify anatomical regions that are significantly different between different populations. This technique was applied to identify such regions between normals controls and two groups of patients with memory impairments, namely subjects who subsequently converted to Alzheimer's Disease and subjects with a mild form of Alzheimer's Disease. Our method clearly identified brain regions known to be affected by this disease. It also singled-out small cortical regions mostly in the posterior part of the brain. Further analysis need to be performed to relate these last findings to clinical data.

Aside from validating and applying this technology to other diseases such as schizophrenia and multiple sclerosis, future work will focus on registration aspects to deal with white matter signal abnormalities present in MR scans obtained from diseased patients, and on the incorporation of spatial information into our statistical model.

\section{References}

1. Mazziotta, J.C., Toga, A.W., Evans, A., Fox, P., Lancaster, J.: A probabilistic atlas of the human brain: Theory and rationale for its development. Neuroimage 2 (1995) 89-101

2. Talairach, J., Tournoux, P.: Co-Planar Stereotaxic Atlas of the Human Brain. Thieme Medical Publishers, New York (1988)

3. Schaltenbrand, G., Wahren, W.: Atlas of Stereotaxy of the Human Brain. Georg Thieme Verlag, Stuttgart (1977)

4. Bajcsy, R., Kovačič, S.: Multiresolution elastic matching. Computer Vision, Graphics and Image Processing 46 (1989) 1-21

5. Greitz, T., Bohm, C., Holte, S., Eriksson, L.: A computerized brain atlas: Construction, anatomical content, and some applications. Journal of Computer Assisted Tomography $\mathbf{1 5}$ (1991) 26-38

6. Lemoine, D., Barillot, C., Gibaud, B., Pasqualini, E.: An anatomical-based 3D registration system of multimodality and atlas data in neurosurgery. Lecture Notes in Computer Science 511 (1991) 154-164

7. Höhne, K.H., Bomans, M., Riemer, M., Schubert, R., Tiede, U., Lierse, W.: A 3D anatomical atlas based on a volume model. IEEE Computer Graphics and Applications 12 (1992) 72-78

8. Evans, A.C., Kamber, M., Collins, D.L., Macdonald, D.: An MRI-based probabilistic atlas of neuroanatomy. In Shorvon, S., Fish, D., Andermann, F., Bydder, G.M., Stefan, H., eds.: Magnetic Resonance Scanning and Epilepsy. (1994) 263-274

9. Bookstein, F.L.: Landmarks, Edges, Morphometrics, and the Brain Atlas Problem. In: Functional Neuroimaging. Academic Press (1994)

10. Christensen, G., Miller, M.I., Vannier, M.W.: A 3D deformable magnetic resonance textbook based on elasticity. In: Spring Symposium: Applications of Computer Vision in Medical Image Processing, Stanford (CA), USA, American Association for Artificial Intelligence (1994)

11. Kikinis, R., Shenton, M.E., Iosifescu, D.V., McCarley, R.W., Saiviroonporn, P., Hokama, H.H., Robatino, A., Metcal, D., Wible, C.G., Portas, C.M., Donnino, R.M., Jolesz, F.A.: A digital brain atlas for surgical planning, model-driven segmentation, and teaching. IEEE Transactions on Visualization and Computer Graphics 2 (1996) 232-241 
12. Le Briquer, L., Gee, J.C.: Design of a statistical model of brain shape. In Duncan, J.S., Gindi, G.R., eds.: Proceedings of the IPMI (IPMI'97), Vermont, USA, Springer-Verlag (1997)

13. Woods, R.P., Grafton, S.T., Watson, J.D.G., Sicotte, N.L., Mazziotta, J.C.: Automated image registration: Ii. intersubject validation of linear and nonlinear models. Journal of Computer Assisted Tomography 22 (1998) 153-165

14. Grenander, U., Miller, M.I.: Computational anatomy: An emerging discipline. Quarterly of Applied Mathematics 56 (1998) 617-694

15. Thompson, P.M., MacDonald, D., Mega, M.S., Holmes, C.J., Evans, A.C., Toga, A.W.: Detection and mapping of abnormal brain structure with a probabilistic atlas of cortical surfaces. Journal of Computer Assisted Tomography 21 (1998) 567-581

16. Subsol, G., Thirion, J.P., Ayache, N.: A scheme for automatically building three-dimensional morphometric anatomical atlases: Application to a skull atlas. Medical Image Analysis 2 (1998) 37-60

17. Guimond, A., Meunier, J., Thirion, J.P.: Average brain models: A convergence study. Computer Vision and Image Understanding 77 (1999) 192-210

18. Gee, J.C., Haynor, D.R., Briquer, L.L., Bajcsy, R.K.: Advances in elastic matching theory and its implementation. In Cinquin, P., Kikinis, R., Lavallee, S., eds.: Proceedings of CVRMed-MRCAS, Heidelberg, Springer-Verlag (1997)

19. Thompson, P.M., Toga, A.W.: Detection, visualization and animation of abnormal anatomic structure with a deformable probabilistic brain atlas based on random vector field transformations. Medical Image Analysis 1 (1997) 271-294

20. Killiany, R.J., Gomez-Isla, T., Moss, M., Kikinis, R., Sandor, T., Jolesz, F.A., Tanzi, R., Jones, K., Hyman, B.T., Albert, M.S.: Use of structural magnetic resonance imaging to predict who will get alzheimer's disease. Annals of Neurology 47 (2000) 430-439

21. Roche, A.: Recalage d'images médicales par inférence statistique. $\mathrm{PhD}$ thesis, Université de Nice Sophia-Antipolis (2001)

22. Roche, A., Malandain, G., Pennec, X., Ayache, N.: The correlation ratio as a new similarity measure for multimodal image registration. In Wells, W.M., Colchester, A., Delp, S., eds.: Proceedings of MICCAI. (1998) 1115-1124

23. Guimond, A., Roche, A., Ayache, N., Meunier, J.: Three-dimensional multimodal brain warping using the demons algorithm and adaptive intensity corrections. IEEE Transactions in Medical Imaging 20 (2001) 58-69

24. Thirion, J.P.: Image matching as a diffusion process: an analogy with Maxwell's demons. Medical Image Analysis 2 (1998) 243-260

25. Anderson, T.W.: An Introduction to Multivariate Statistical Analysis. Second edn. Probability and Mathematical Statistics. John Wiley \& Sons (1984)

26. Fukunaga, K.: Introduction to Statistical Pattern Recognition. Second edn. Computer science and scientific computing. Academic Press (1990)

27. Hughes, C.P., Berg, L., Danziger, W.L., Coben, L., Martin, R.: A new clinical scale for the staging of dementia. British Journal of Psychiatry 140 (1982) 566-572

28. McKhann, G., Drachman, D., Folstein, M., Kaltzman, R., Price, D., Stadlan, E.: Clinical diagnosis of alzheimer's disease: Report of the nincds-adrda work group under the auspices of department of health and human services task force on alzheimer's disease. Neurology 39 (1984) 939-944

29. Kemper, T.L.: Neuroanatomical and Neuropathological Changes in Normal Aging and in Dementia. In: Clinical Neurology of Aging. Oxford University Press, New-York (1984) 9-52

30. Guimond, A., Wei, X., Guttmann, C.R.G.: Building a probabilistic anatomical brain atlas for multiple sclerosis. In: Proceedings of ISMRM. (2002) In Print.

31. Kapur, T.: Model based three dimensional Medical Imaging Segmentation. PhD thesis, Massachusetts Institute of Technology (1999) 